\title{
Diagnostic potential of ultrasound in carpal tunnel syndrome with different etiologies: correlation of sonographic median nerve measures with electrodiagnostic severity
}

Basant Elnady ${ }^{1,2^{*}}$ (D) Elsayed M. Rageh ${ }^{3}$, Tohamy Ekhouly ${ }^{4,5}$, Sabry M. Fathy ${ }^{6,7}$, Mohamed Alshaar ${ }^{6,7}$, El Saeed Fouda ${ }^{8}$, Mohammed Attar ${ }^{2}$, Ahmed M. Abdelaal ${ }^{9}$, Ahmed El Tantawi ${ }^{10,11}$, Mohammed M. Algethami ${ }^{2}$ and David Bong ${ }^{12}$

\begin{abstract}
Background: Carpal tunnel syndrome (CTS) is the commonest entrapment neuropathy. The aim of this study was to assess the accuracy and validity of high resolution musculoskeletal ultrasound (US) in the diagnosis of CTS in the Saudi population.

Methods: Sixty patients were diagnosed clinically to have CTS involving 89 wrists that were confirmed by neurophysiologic studies. Each affected wrist was characterized as idiopathic or associated with either diabetes mellitus or hypothyroidism and were assigned a severity grade based on results of neurophysiologic studies. Seventy-six healthy wrists from fifty age, sex and BMI matched healthy subjects were included in the control group. High resolution ultrasound (US) was performed to assess median nerve cross sectional area distal (CSAd) at the entry to the carpal tunnel and proximally (CSAp) at the level of pronator quadratus muscle with a further calculation of their difference $(\triangle C S A)$ and their mean average or CSAd+CSAp/2 (CSApd).

Results: There was a significant difference between both groups regarding mean \pm SD of CSAd, CSAp, $\triangle$ CSA, and CSApd $(p=0.0001)$. A positive significant correlation was also found between the CSAd, $\triangle$ CSA and the CSApd measurements with neurophysiologic severity grade of CTS $(P=0.001)$. A $\triangle C S A$ threshold of $2.5 \mathrm{~mm}^{2}$ showed the highest sensitivity and specificity to diagnose CTS in Saudis.
\end{abstract}

Conclusion: High resolution ultrasound is a valid and accurate diagnostic modality in carpal tunnel syndrome and correlated to CTS severity. A $\triangle$ CSA greater than $2.5 \mathrm{~mm}^{2}$ is considered a valid diagnostic value for CTS in our Saudi population. CTS in our patients with diabetes tend to have greater median nerve US measurement values.

Keywords: Carpal tunnel syndrome, Median nerve, Ultrasound

\section{Background}

Carpal tunnel syndrome (CTS) is the most frequent nerve entrapment neuropathy; it occurs secondary to compression of the median nerve under the flexor retinaculum of wrist joint and leads to an enlargement of its

\footnotetext{
* Correspondence: basantelnady@gmail.com

'Department of Rheumatology, Rehabilitation and Physical Medicine, Benha University, Benha, Egypt

²Department of Internal Medicine, Al Hada Forces Hospital, Alhada, Saudi Arabia

Full list of author information is available at the end of the article
}

cross-sectional area (CSA) just proximal to the site of entrapment (1).

CTS is considered an idiopathic condition, however, characteristic anatomical variations may participate in development of CTS, such as persistent median artery, or a bifid median nerve (2). It may also result from traumatic injury, inflammatory arthritis such as rheumatoid arthritis, or in association of hypothyroidism, diabetes mellitus, or pregnancy $(3,4)$.

Early diagnosis is essential to alleviate permanent nerve damage and functional disability. The diagnosis of 
CTS is usually based on clinical and neurophysiological studies (1).

US is a diagnostic imaging modality is being used more often in daily clinical practice not only to confirm the diagnosis of CTS but also it can detect anatomical variations, nerve shape and space-occupying lesions as tenosynovitis and ganglion cysts $(5,6)$.

Modern US equipment allows freehand tracing of the circumference of the median nerve proximal to the site of entrapment and calculation of the cross-sectional area (CSA). Unfortunately, there is no clear cut and generally accepted cutoff value of a single CSA measurement to diagnose CTS as different studies have producing widely variable results with various cutoff threshold for establishing the diagnosis of CTS which appear to be affected by group ethnicity. (7-16). Although studies had investigated median nerve CSA at the wrist in CTS patients exclusively with diabetes and hypothyroidism (17-19), a comparison of different US parameters, especially the CSA, in patients with idiopathic CTS versus those with CTS associated with diabetes and hypothyroidism has not been compared in the same study.

Klauser et al., assessed the CSA of median nerve distally at the region of maximal enlargement, usually just proximal to the carpal tunnel, and proximally at the level of pronator quadratus muscle, thereby using the same nerve as its own control, to improve the precision of CTS diagnosis (20).

The aim of this study was to assess the accuracy of ultrasound in diagnosis of CTS in the Saudi population using the method developed by Klauser et al. (i.e. $\triangle$ CSA) and also the mean of the proximal and distal (mean CSApd) measurements. The median nerve US measurements were further analyzed to detect any difference in the parameters between idiopathic CTS and CTS associated with diabetes mellitus or hypothyroidism.

\section{Methods \\ Study design \\ Cross sectional cohort, randomized study}

Study population Sixty consecutive electrophysiologically confirmed CTS patients presenting to a tertiary Saudi medical center (Al Hada Armed Forces Hospital) rheumatology, neurology, neurosurgery out-patients' clinics, during a six month period underwent US evaluation and fifty age, sex and body mass index (BMI) matched healthy subjects free from CTS manifestation with negative electrophysiologic studies for CTS from the healthy hospital workers were included as a control group for identical US study.

Inclusion criteria Age $>18$ years; right handed patients with paresthesia, numbness or tingling affecting the first three digits and the radial half of the fourth digit, which was confirmed by electrophysiologic study.
Exclusion criteria $B M I \geq 30$, past history of traumatic or surgical intervention; arthritis; median nerve injection, history of autoimmune rheumatic disease; bifid median nerve, or intra-articular lesions such as ganglion. Patients with nerve conduction study (NCS) findings suggestive of diabetic neuropathy rather than entrapment neuropathy at the carpal tunnel also were excluded.

\section{Electrophysiologic methods}

The electrodiagnostic studies were performed according to standard techniques for motor and sensory nerve conduction studies of median and ulnar nerves. Motor study included the determination of conduction velocity, amplitudes and latencies after stimulation of the median nerve. Sensory nerve conduction studies included the antidromic determination of conduction velocity, latencies and amplitude of the sensory nerve action potential of the median nerve. These studies were performed for all participants within 2 weeks before US examination by using a Dantec Key Point electrophysiologic device at a fixed room temperature of $25^{\circ} \mathrm{C}$ during the examination of all patients and controls.

Diagnosis of CTS was based on the measurement of the median nerve compound muscle action potential (CMAP) amplitude and distal latency from the abductor pollicis brevis following stimulation $8 \mathrm{~cm}$ proximal to the recording electrode and the sensory nerve action potential (SNAP) obtained from the middle finger with ringtype electrodes. The palmar and wrist stimulations were 7 and $14 \mathrm{~cm}$, respectively, proximal to the recording electrode.

Electrodiagnostic studies were done on both hands by expert neurologist blinded to the clinical data and study purpose. Only patients with three of the following criteria were included: median SNAP peak latency $>3.7 \mathrm{~ms}$; a SNAP peak latency of the proximal $7-\mathrm{cm}$ segment greater than the peak latency of the distal 7-cm segment; SNAP amplitude was $<20 \mu \mathrm{V}$ including a conduction block (a SNAP amplitude drop of $>50 \%$ with respect to the proximal stimulation, as compared with that of the distal stimulation); median CMAP distal latency was > $4.2 \mathrm{~ms}$; and CMAP amplitude was $<4.5 \mathrm{mV}$. CTS was diagnosed in patients with diabetic neuropathy if they met the following criteria: the ratio of the distal motor latency of the median to the ulnar nerve was $>1.5$; the ratio of the distal sensory latency of the median to the ulnar nerve was $>1.2$; the amplitude ratio of the median SNAP to the ulnar SNAP was $<0.6(21-25)$.

The severity of CTS was classified as mild, moderate or severe according to the modified scoring system of Padua et al. (26); Severe (absence of sensory response, abnormal distal motor latency (DML)), Moderate (abnormal sensory nerve conduction velocity (SNCV), abnormal DML), Mild (abnormal SNCV, normal DML). 
Furthermore, electrophysiologically confirmed CTS patients were stratified as to the etiology: idiopathic, diabetic or hypothyroid.

\section{US technique}

Sixty consecutive patients with electrophysiologically confirmed CTS and 50 healthy controls with normal electrophysiologic studies of the median nerve were examined by US by two investigators expert radiologist and rheumatologist blinded to the clinical and electrophysiologic results. 89 out of 120 wrists of the patients fulfilled inclusion criteria and were evaluated by US, whereas 76 of 100 wrists of the healthy controls underwent US evaluation (Philips CX50 scanner, using two multi-frequency linear transducers, $6-18 \mathrm{MHz}$ and 5-12 $\mathrm{MHz}$ ). Transverse US scanning of the median nerve from the distal forearm to the carpal tunnel outlet was performed with measurement of the median nerve CSA distal (CSAd) at its apparent maximal dimension of the thickest part of median nerve at the tunnel (Figs. 1, 2, 3a). Proximal transverse US scanning for CSA proximal (CSAp) measurement was done over the distal third of the pronator quadratus muscle (Figs. 1, 2, 3b). The median nerve was identified between the flexor digitorum superficialis and flexor pollicis longus muscle/myotendinous junction. Then the difference between CSAd and CSAp ( $\triangle$ CSA) was calculated for each wrist in both patients and controls (20). A mean of the two CSA's on each US study was calculated by adding the tabulated values of CSAd and CSAp and dividing the sum by two to get the mean CSAdp (CSAd+CSAp/2).

We used the direct measurement technique, after capturing the appropriate image. Free-hand tracing was done with a continuous line around the median nerve fascicules excluding the outer hyperechoic rim (epineurium/fat). These measurements were obtained three times at both CSAd and CSAp levels and a median value for each site was recorded for statistical analysis (27).

\section{Statistical analysis}

The Quantitative data were expressed as mean and standard deviation (Mean $\pm \mathrm{SD}$ ), Inter-reader agreement on ultrasound measurements was investigated using Cohen's kappa coefficient between the two ultrasonography readings. For the analytic purpose there was no significant difference between CSAd, CSAp, $\triangle$ CSA and Mean CSApd, $(p>0.05)$ measurements in the right and left wrists measurements of patients with bilateral CTS, so each wrist assumed as independent variable for analysis. The CTS group and healthy controls group were compared using a $t$ test for age, CSAd, CSAp, mean CSAdp and the $\triangle$ CSA measurements. $\chi^{2}$ test for subject sex. The comparison in relation to neurophysiologic severity the CSAd, mean CSAdp and the $\triangle$ CSA were compared using a $t$ test. Pearson correlation coefficients were computed for measurements of CSAd, mean CSAdp and $\triangle$ CSA to determine whether there was any significant correlation between measurements obtained from the right and left wrist measurements in patients with bilateral CTS evaluation, also correlation coefficients were done to assess the relation of CSAd, mean CSAdp and $\triangle$ CSA measurements with the median nerve neurophysiological grades of severity.

The accuracy and cutoff values of US in the diagnosis of CTS were tabulated by using a receiver operating characteristic (ROC) analysis for CSAd, mean CSAdp and $\triangle$ CSA measurements.

Analysis of variance (ANOVA) was used to test the differences in $\triangle$ CSA measurements in relation to different CTS etiologies (idiopathic, diabetes and hypothyroidism). All statistical tests were performed with (SPSS) version 20 (Armonk, NY: IBM Corp.), and $P<0.05$ indicated a significant difference.

\section{Ethics}

The study conforms to the 1995 Helsinki declaration and was approved by the ethical committee of $\mathrm{Al} \mathrm{Hada}$ Armed Forces Hospital, KSA. Written consent form was taken from all patients prior to their inclusion.

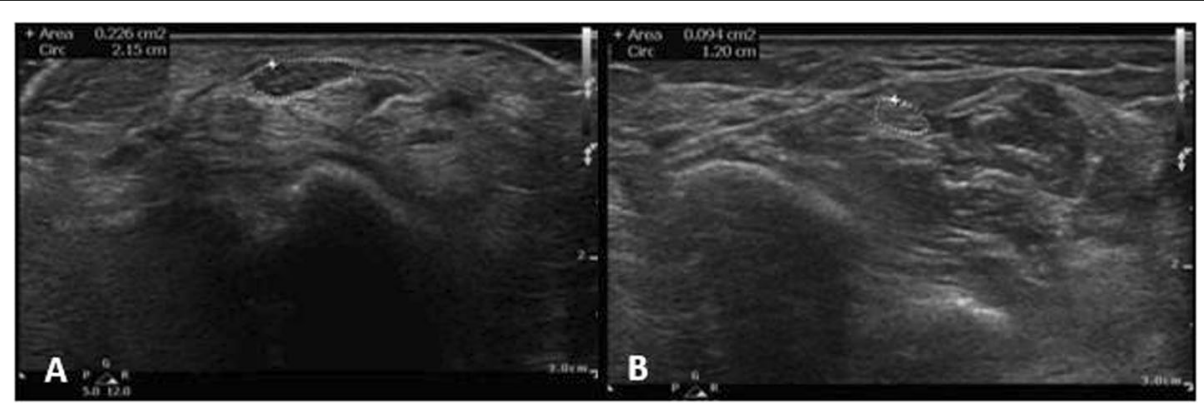

Fig. 1 Transverse US image of right median nerve (outlined) at level of carpal tunnel (A) with CSAd is $22.6 \mathrm{~mm}^{2}$ and at the level of pronator quadratus muscle(B) with CSAp is $9.4 \mathrm{~mm}^{2}$ in a patient with diabetes and CTS 


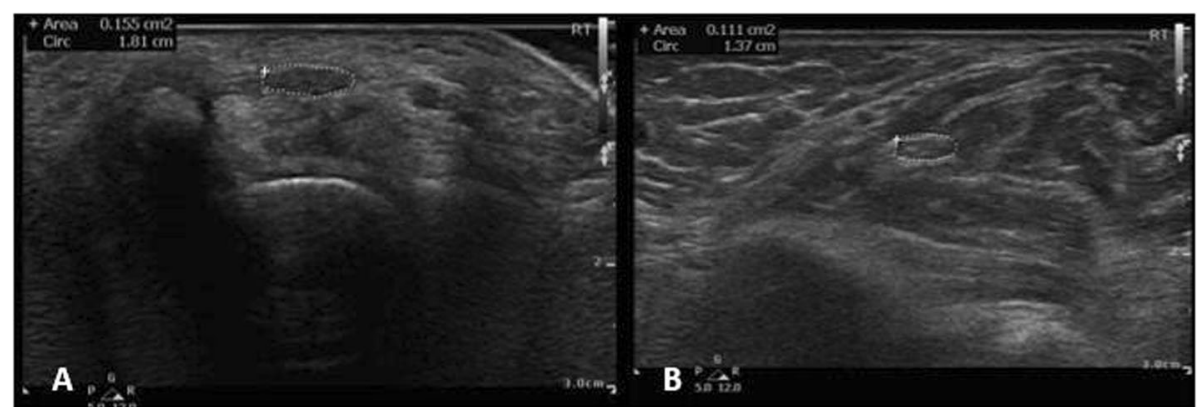

Fig. 2 Transverse US image of right median nerve (outlined) at level of carpal tunnel (A) with CSAd is $15.5 \mathrm{~mm}^{2}$ and level of pronator quadratus muscle(B) with CSAp is $11.1 \mathrm{~mm}^{2}$ in a patient with hypothyroidism and CTS

\section{Results}

42/60(70\%) patients were females and 38/50 (76\%) controls were females. The mean patient age was $50.2 \pm$ 11.2 years. 89 wrists of the 60 CTS patients and 76 wrists of the 50 healthy controls were scanned by US,

There was no statistical significant difference regarding age, sex and BMI between CTS patients and controls. However there was a highly significant difference between both groups regarding mean \pm SD of CSAd, CSAp, $\triangle$ CSA, and mean CSApd ( $p=0.0001)$ (Table 1).

Severe CTS according to neurophysiological assessment was found in 31 wrists with 58 wrists diagnosed as mild to moderate. Cohen's kappa test showed highly statistical inter-reader agreement regarding both ultrasonography measurements $(\mathrm{k}=0.88, p<$ 0.001 ). There was a statistical significant difference in CSAd values between mild to moderate CTS (16.4 \pm $\left.5.1 \mathrm{~mm}^{2}\right)$ and severe CTS $\left(19.7 \pm 5.3 \mathrm{~mm}^{2}\right) \quad(P=0.01)$, additionally there was a highly statistical significant difference in $\triangle \mathrm{CSA}$ values between mild to moderate CTS $\left(5.8 \pm 3.1 \mathrm{~mm}^{2}\right)$ and severe CTS $\left(11 \pm 4.3 \mathrm{~mm}^{2}\right)$ $(P=0.001)$, furthermore there was a highly statistical significant difference in the mean of CSApd values between mild to moderate CTS $\left(8.8 \pm 1.5 \mathrm{~mm}^{2}\right)$ and severe CTS $\left(14.4 \pm 4.2 \mathrm{~mm}^{2}\right)$.
A positive significant correlation was also found between the CSAd, $\triangle$ CSA and mean of CSApd measurements with the severity grade based on the neurophysiologic studies $(\mathrm{P}=0.001)$ (Table 2).

To assess the accurate cutoff value and the most sensitive and specific threshold values, a receiver operating characteristic (ROC) curve was done and the area under the curve was calculated. The ultrasound measures of CSAd with area under the curve $=0.978$, showed threshold values of 11,12 , and $13 \mathrm{~mm}^{2}$, the $\triangle$ CSA with area under the curve $=0.998$, showed threshold values of 2 , 2.5, $3 \mathrm{~mm}^{2}$, and mean CSAdp with area under the curve $=0.925$, showed threshold values of 9.5, 10, 10.5 $\mathrm{mm}^{2}$ were illustrated in Table 3 . The best diagnostic values were achieved by using a $\triangle$ CSA threshold of 2.5 $\mathrm{mm}^{2}$. Receiver operating characteristic analysis revealed excellent ability to use CSAd, $\triangle$ CSA, and Mean CSAdp (Fig. 4, Table 3).

58 scanned CTS wrists were characterized as idiopathic, 18 diabetes-associated CTS and 13 hypothyroidism-associated CTS. There was no significant difference regarding $\triangle \mathrm{CSA}$ between diabetic, idiopathic and hypothyroidism CTS groups $(P>0.05)$, however there was statistical significant difference regarding mean CSAdp and CSAd values within groups

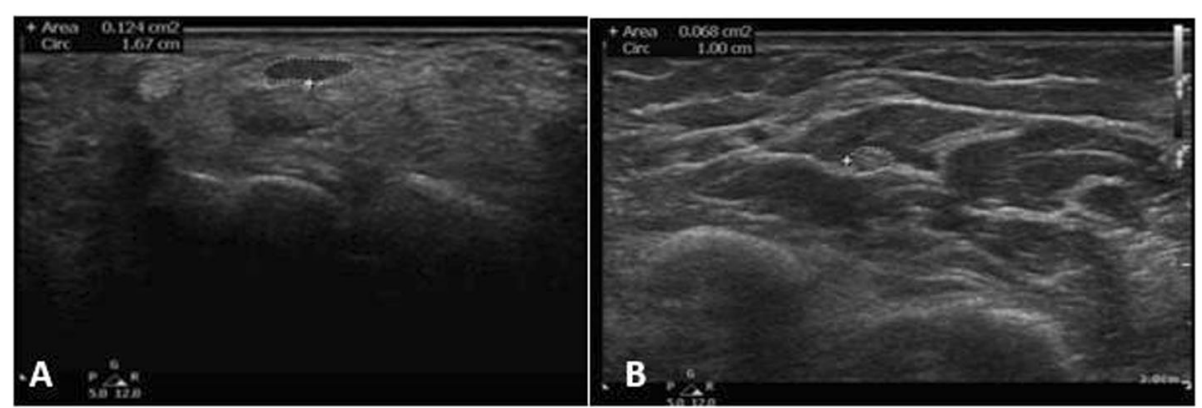

Fig. 3 Transverse US image of right median nerve (outlined) at level of carpal tunnel (A) with CSAd is $12.4 \mathrm{~mm}^{2}$ and level of pronator quadratus muscle(B) with CSAp is $6.8 \mathrm{~mm}^{2}$ in a patient with idiopathic CTS 
Table 1 Demographic characters of the studied group

\begin{tabular}{llll}
\hline Variable & CTS patients number of scanned wrists (89) & Control group number of scanned wrists(76) & $P$-value \\
\hline Sex $\%$ & $42 / 60(70 \%)$ & $38 / 50(76 \%)$ & 0.14 \\
Age Mean and SD & $50.2 \pm 11.2$ years & $48.4 \pm 10.2$ years & 0.8 \\
BMI kg/m & $25.4 \pm 4.8$ & $24.3 \pm 4.6$ & 0.2 \\
CSAd Mean and SD & $18.4 \pm 5.4$ & $9.3 \pm 1.6$ & 0.0001 \\
CSAp Mean and SD & $9.3 \pm 2.7$ & $8.4 \pm 1.6$ & 0.01 \\
$\triangle$ CSA Mean and SD & $9.2 \pm 4.6 \mathrm{~mm} 2$ & $0.9 \pm 1.5 \mathrm{~mm} 2$ & 0.0001 \\
Mean CSAdp & $14.5 \pm 4.2 \mathrm{~mm} 2$ & $8.8 \pm 1.5 \mathrm{~mm} 2$ & 0.0001
\end{tabular}

Carpal tunnel syndrome (CTS), cross sectional area distal (CSAd), cross sectional area proximally (CSAp), cross sectional area difference ( $\triangle$ CSA), mean average of cross sectional area distal and proximal (CSApd), body mass index (BMI), standard deviation (SD), $P$ value was calculated by independent $\mathrm{t}$-test between two groups with $95 \% \mathrm{Cl}$

$(P<0.05)$, Post Hoc analysis was done showed no significant difference regarding mean CSAdp and CSAd values between idiopathic and hypothyroidism associated CTS or between hypothyroid and diabetes associated CTS. However a significant difference was found between mean CSAdp and CSAd values between diabetes and idiopathic CTS, with higher measurement values in diabetic patients $(\mathrm{P}<0.05)$ (Table 4$)$.

\section{Discussion}

Median nerve entrapment in the wrist joint is the most frequent entrapment neuropathy [1, 28], with high prevalence in females [29]. US is a noninvasive tool that can assess the median nerve in CTS [5, 6]. We investigated the use of US CSA measurements of the median nerve in patients with electrophysiological confirmed CTS and in non-affected normal controls to validate this technique in the Saudi population. We evaluated the difference and average mean of both the proximal, non affected, part of the median nerve at the level of the pronator quadratus muscle and the CSA at the wrist where you would expect to find the maximum enlargement due to distal compression effect, [20]. Furthermore, we calculated the mean of CSA proximal values to omit the individual variability rather than depend on CSA at the level of carpal tunnel only.

We found a statistical significant difference in the US values of CSAd, CSAp, $\triangle$ CSA, and mean CSAdp between CTS patients and healthy controls, with these findings was in agreement with Klauser et al. [20].

Table 2 Correlation of CTS neurophysiological Severity in Nerve Conduction Examination and ultrasound measurement

\begin{tabular}{lll}
\hline Correlation with NCS grade of severity & $r$ & $p$-value \\
\hline CSAd & 0.278 & 0.008 \\
$\triangle$ CSA Mean and SD & 0.644 & 0.0001 \\
Mean CSAdp & 0.293 & 0.005 \\
\hline
\end{tabular}

Cross sectional area distal (CSAd), cross sectional area difference $(\triangle C S A)$, mean average of cross sectional area distal and proximal (CSApd), nerve conduction study (NCS), P value was calculated by Pearson correlation test
Although neurophysiologic study is accurate in diagnosis of CTS [1], US has the added value of potentially identifying the underlying pathology causing CTS, such as anatomical variants or space occupying pathology [36]. In the our study, the CSAd was statistically correlated to the severity of CTS with neurophysiologic assessment, the higher the value the more severe the CTS, which were concordant with other studies [20, 30, 31]. However, Mhoon et al. did not find a significant correlation between CSAd and electrophysiologic severity assessment [32]. Klauser et al. also [20] stated that $\triangle$ CSA is correlated with neurophysiologic CTS severity, which agreed with our findings related to $\triangle$ CSA. In addition, we found that the mean CSAdp also statistically correlated to the severity of CTS which was not assessed in previous studies.

Previous studies attempted to ascertain a universal cutoff value of CSA for the diagnosis of CTS, however until now there was no standard universal cutoff range, being mainly dependent on the ethnicity of the group studied [7-12]. In previous literature, the suggested values of CSAd median nerve abnormality varies between 9 to $14 \mathrm{~mm}^{2}[27,28]$. Although discrepancies in the accuracy of sonographic criteria of median nerve

Table 3 Sensitivity and Specificity of Nerve Measurements in the Diagnosis of CTS

\begin{tabular}{lll}
\hline Measurement & Sensitivity (\%) & Specificity (\%) \\
\hline CSAd $11 \mathrm{~mm} 2$ threshold & 97 & 89 \\
CSAd $12 \mathrm{~mm} 2$ threshold & 92 & 94 \\
CSAd 13 mm2 threshold & 90 & 95 \\
$\triangle$ CSA with 2 -mm2 threshold & 97 & 90 \\
$\triangle$ CSA with 2.5-mm2 threshold & 97 & 100 \\
$\triangle$ CSA with 3-mm2 threshold & 96 & 100 \\
Mean CSAdp with 9.5-mm2 threshold & 90 & 81 \\
Mean CSAdp with 10 -mm2 threshold & 83 & 85 \\
Mean CSAdp with 10.5-mm2 threshold & 82 & 90 \\
\hline
\end{tabular}

Cross sectional area distal (CSAd), cross sectional area difference $(\triangle \mathrm{CSA})$, mean average of cross sectional area distal and proximal (CSApd), 

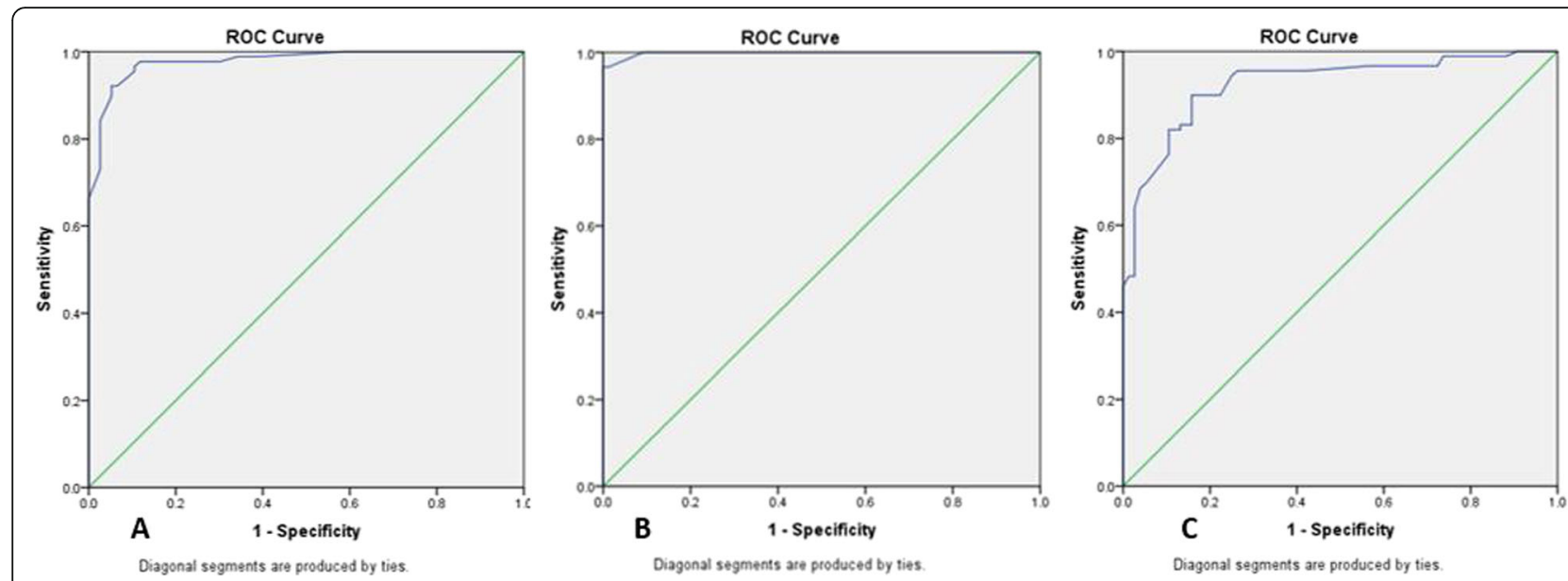

Fig. 4 The receiver operating characteristic (ROC) curve for CSAd (A), $\triangle C S A(B)$ and Mean CSAdp (C) measurements in CTS patients

entrapment have been reported, CSA with cutoff values of more than 9 or $10 \mathrm{~mm}^{2}$ at the scapho-pisiform level indicating CTS [12-15] is still agreed upon as the most reliable and reproducible sonographic criterion indicating CTS [33], with sensitivity of $82 \%$ and specificity of $87 \%$ (which were almost equal to those of electrodiagnostic values) with a cutoff CSA of more than $12 \mathrm{~mm}^{2}$ considered as excellent to diagnose CTS [11]. In the current work, the cutoff value of CSA at the level of

Table 4 Comparative study of CTS ultrasonography measurement in relation to different etiology

\begin{tabular}{lcll}
\hline Ultrasound measurement & Mean and SD & Etiology & P-value \\
\hline Idiopathic CSAd & $17.59 \pm 4.84$ & Diabetes & $\mathbf{0 . 0 0 2}$ \\
& & Hypothyroidism & 0.6 \\
Diabetes CSAd & $22.01 \pm 6.5$ & $\begin{array}{l}\text { Idiopathic } \\
\text { Hypothyroidism }\end{array}$ & $\mathbf{0 . 0 0 2}$ \\
Hypothyroidism CSAd & $18.5 \pm 4.47$ & Idiopathic & 0.057 \\
& & Diabetes & 0.07 \\
Idiopathic $\triangle$ CSA & $8.52 \pm 4.55$ & Diabetes & 0.08 \\
& & Hypothyroidism & 0.5 \\
Diabetes $\triangle$ CSA & $11.2 \pm 5.1$ & Idiopathic & 0.08 \\
& & Hypothyroidism & 0.3 \\
Hypothyroidism $\triangle$ CSA & $9.48 \pm 3.75$ & Idiopathic & 0.5 \\
& & Diabetes & 0.3 \\
Idiopathic Mean CSAdp & $13.57 \pm 3.37$ & Diabetes & $\mathbf{0 . 0 2}$ \\
& & Hypothyroidism & 0.07 \\
Diabetes Mean CSAdp & $16.48 \pm 5.24$ & Idiopathic & $\mathbf{0 . 0 2}$ \\
& & Hypothyroidism & 0.6 \\
Hypothyroidism Mean CSAdp & $15.76 \pm 4.67$ & Idiopathic & 0.07 \\
& & Diabetes & 0.6 \\
\hline
\end{tabular}

Cross sectional area distal (CSAd), cross sectional area difference $(\triangle C S A)$, mean average of cross sectional area distal and proximal (CSApd), $P$ value was calculated by ANOVA test carpal tunnel to diagnose CTS in Saudi population was $13 \mathrm{~mm}^{2}$ with $90 \%$ sensitivity and $95 \%$ specificity which was in agreement with other studies cutoff threshold that ranged between 12 and $13 \mathrm{~mm}^{2}$ in CTS with or without diabetes $[20,34,35]$. The mean CSAdp of 9.5 showed sensitivity of 82 and $95 \%$ specificity to diagnose CTS, however $\triangle$ CSA of $2.5 \mathrm{~mm}^{2}$ was associated with 97\% sensitivity and 100\% specificity, with much higher sensitivity and specificity than using CSAd and mean CSAdp. In another study, the $\triangle$ CSA of $2 \mathrm{~mm}^{2}$ or greater was considered diagnostic for CTS with sensitivity of $99 \%$ and a specificity of $100 \%$ [20].

Electrodiagnostic testing is considered the reference standard for CTS diagnosis with sensitivities of $82-94 \%$ and specificities of $65-97 \%$ [10-12, 15], even though paresthesia may occur before changes can be measured with nerve conduction tests [36]. US is advantageous in that it is noninvasive, less painful (and therefore more acceptable to patients), more readily available and less expensive. According to our results, US is highly sensitive and specific, so that it may be used as an early tool to assess CTS in patients that may have negative or borderline electrophysiologic studies since negative electrophysiologic studies may occur in up to $30 \%$ of CTS patients [9]. According to our results, ultrasound is highly sensitive and specific, so it may be used as an early tool to assess CTS where patients may have negative or borderline electrophysiologic studies.

In the current study, we also looked at different CTS etiologies. There was a statistical difference regarding CSAd, and mean CSAdp values between CTS patients with idiopathic CTS and diabetes, however, there was no statistical difference between different etiologic groups regarding $\triangle$ CSA. A recent meta-analysis on CSA of CTS with diabetes, in spite being not statistically significant $(p=0.52)$, CSA of median nerve in patients with diabetes and CTS were likely to be larger than patients with 
idiopathic CTS [17]. A study by Thomsen et al. found focal CSA enlargement of median nerve in diabetic patients without CTS, particularly at the level of the carpal tunnel inlet, they suggested other additional factors which may contribute to such phenomenon, such as a reduction in myelinated nerve fibers and capillary density that may predispose diabetics to develop CTS [37]. Imaging modalities as US and magnetic resonance imaging (MRI) have had a significant role in understanding, assessment and diagnosis of disorders of the peripheral nervous disorders [39].

Our study had several limitations. The assessment of US measurements CSAd, $\triangle$ CSA and mean CSAdp in cases with bifid median was not done on our group of patients. In addition, this study is only assessed in one ethnic group with smaller sample size.

Strengths of the current study are that it was designed as a case controlled study involving a less commonly studied ethnic group, the inclusion of disease severity and various etiologies, the correlation of the different US measurements to disease severity, the sub analysis of US measurements in relation to variable etiologies and the establishment of a cutoff diagnostic value of CTS in Saudi population with ultrasound.

\section{Conclusion}

Ultrasound assessment in carpal tunnel syndrome is noninvasive diagnostic tool with correlation to the degree of entrapment severity with a $\triangle$ CSA greater than $2.5 \mathrm{~mm}^{2}$ being considered diagnostic for CTS in Saudi population. Diabetic CTS patients tend to have higher median nerve US measurements values as opposed to idiopathic and hypothyroid CTS in our study group. This study, in our opinion, emphasizes the importance of utilizing US in the diagnosis of early stage CTS to alleviate permanent nerve damage and functional disability, however, large randomized control studies are needed to assess the current study measurements in bifid median nerve with CTS.

\footnotetext{
Abbreviations

BMI: Body mass index; CMAP: Compound muscle action potential; CSAd: Cross sectional area distal; CSAp: Cross sectional area proximally; CSApd: Mean average of cross sectional area distal and proximal; CTS: Carpal tunnel syndrome; DML: Distal motor latency; MRI: Magnetic resonance imaging; NCS: Nerve conduction study; ROC: Receiver operating characteristic; SD: Standard deviation; SNAP: Sensory nerve action potential; SNCV: Sensory nerve conduction velocity; US: Ultrasound; $\triangle C S A$ : Cross sectional area difference
}

\section{Acknowledgements}

The authors gratefully acknowledge the support provided by the officials of the studied hospital for facilitating the administrative aspects of the research. Special thanks to the participant patients for their cooperation.

\section{Author's contribution}

BE: study design, ultrasound examination and authoring; ER: references collection, data interpretation and authoring; TE: ultrasound examination; SF: data and references collection, performing nerve conduction study; MA: performing nerve conduction study; EF: data analysis; MAA:recruiting patient with clinical examination; AA: authoring and references collection; AT: recruiting patients and examination; MMA: authoring and reference collection; DB: study design, editing and supervision.

All authors read and approved the final manuscript.

\section{Funding}

None.

\section{Availability of data and materials}

The datasets used and/or analysed during the current study are available from the corresponding author on reasonable request.

\section{Ethics approval and consent to participate}

The study conforms to the 1995 Helsinki declaration and was approved by the ethical committee of Al Hada Armed Forces Hospital, KSA. Written consent form was taken from all patients prior to their inclusion.

\section{Consent for publication}

All participants are consented for related radiological images and, clinical data publication.

\section{Competing interests}

The authors declares that they have no competing of interests.

\section{Author details}

${ }^{1}$ Department of Rheumatology, Rehabilitation and Physical Medicine, Benha University, Benha, Egypt. ²Department of Internal Medicine, Al Hada Forces Hospital, Alhada, Saudi Arabia. ${ }^{3}$ Department of Rheumatology, Rehabilitation and Physical Medicine, Tanta University, Tanta, Egypt. ${ }^{4}$ Department of Radiology, Benha University, Benha, Egypt. ${ }^{5}$ Department of Radiology, Al Hada Forces Hospital, Al Hada, Saudi Arabia. ${ }^{6}$ Department of Neurology, Al Azhar University, Al Azhar, Egypt. Department of Neurology, Al Hada Forces Hospital, Alhada, Saudi Arabia. ${ }^{8}$ Department of Pediatrics, Menoufia University, Shebeen El-Kom, Egypt. ${ }^{9}$ Department of Neurosurgery, Salford Royal NHS Foundation Trust, Salford, England. ${ }^{10}$ Department of Neurosurgery, Ain Shams University, Ain Shams, Egypt. ${ }^{11}$ Department of Neurosurgery, Al Hada Forces Hospital, Al Hada, Saudi Arabia. ${ }^{12}$ Instituto Poal de Reumatologia, University of Barcelona, Barcelona, Spain.

Received: 11 October 2019 Accepted: 17 December 2019

Published online: 29 December 2019

\section{References}

1. Gelberman $\mathrm{RH}$, Eaton R, Urbaniak JR. Peripheral nerve compression. Instr Course Lect. 1994;43:31-53.

2. Kotov G, lliev A, Georgiev GP, Karabinov V, Landzhov B. Acta Morphol Anthropol. Clinical significance of anatomical variations in the carpal tunnel: literature review. 2017:24:109-13.

3. Georgiev GP, Karabinov V, Matev B, Iliev A, Kotov G, Landzhov B. Carpal tunnel syndrome treatment with open surgical release: a study in 292 patients. Acta Morphol Anthropol. 2017;24:76-81.

4. Bongers FJ, Schellevis FG, van den Bosch WJ, van der Zee J. Carpal tunnel syndrome in general practice (1987 and 2001): incidence and the role of occupational and non-occupational factors. Br J Gen Pract. 2007:57:36-9.

5. Martinoli C, Bianchi S, Gandolfo N, Valle M, Simonetti S, Derchi LE. US of nerve entrapments in osteofibrous tunnels of the upper and lower limbs. RadioGraphics. 2000:20:199-213.

6. Buchberger W, Judmaier W, Birbamer G, Lener M, Schmidauer C. Carpal tunnel syndrome: diagnosis with high-resolution sonography. AJR Am J Roentgenol. 1992;159(4):793-8.

7. Lee D, van Holsbeeck MT, Janevski PK, Ganos DL, Ditmars DM, Darian VB. Diagnosis of carpal tunnel syndrome: ultrasound versus electromyography. Radiol Clin N Am. 1999;37(4):859-72.

8. Wiesler ER, Chloros GD, Cartwright MS, Smith BP, Rushing J, Walker FO. The use of diagnostic ultrasound in carpal tunnel syndrome. J Hand Surg [Am]. 2006:31(5):726-32.

9. Koyuncuoglu HR, Kutluhan S, Yesildag A, Oyar O, Guler K, Ozden A. The value of ultrasonographic measurement in carpal tunnel syndrome in patients with negative electrodiagnostic tests. Eur J Radiol. 2005;56(3):365-9. 
10. Wong SM, Griffith JF, Hui AC, Lo SK, Fu M, Wong KS. Carpal tunnel syndrome: diagnostic usefulness of sonography. Radiology. 2004;232(1):939.

11. Ziswiler HR, Reichenbach S, Vogelin E, Bachmann LM, Villiger PM, Juni P. Diagnostic value of sonography in patients with suspected carpal tunnel syndrome: a prospective study. Arthritis Rheum. 2005:52(1):304-11.

12. Yesildag A, Kutluhan $\mathrm{S}$, Sengul N, et al. The role of ultrasonographic measurements of the median nerve in the diagnosis of carpal tunnel syndrome. Clin Radiol. 2004;59(10):910-5.

13. Sernik RA, Abicalaf CA, Pimentel BF, Braga-Baiak A, Braga L, Cerri GG. Ultrasound features of carpal tunnel syndrome: a prospective case-control study. Skelet Radiol. 2008;37(1):49-53.

14. Yucel A, Yilmaz O, Babaoglu S, Acar M, Degirmenci B. Sonographic findings of the median nerve and prevalence of carpal tunnel syndrome in patients with Parkinson's disease. Eur J Radiol. 2008;67(3):546-50.

15. Duncan I, Sullivan P, Lomas F. Sonography in the diagnosis of carpal tunnel syndrome. AJR Am J Roentgenol. 1999;173(3):681-4.

16. Buchberger W, Schon G, Strasser K, Jungwirth W. High-resolution ultrasonography of the carpal tunnel. J Ultrasound Med. 1991;10(10):531-7.

17. Chen IJ, Chang KV, Lou YM, Wu WT, Özçakar L. Can ultrasound imaging be used for the diagnosis of carpal tunnel syndrome in diabetic patients? A systemic review and network meta-analysis. J Neurol. 2019 Feb 25. https:// doi.org/10.1007/s00415-019-09254-8.

18. Holováčová D, Kužma M, Killinger Z, Payer J. Cross-sectional area of the median nerve is increased in primary autoimmune hypothyroidism and decreases upon treatment with thyroxine. Eur J Endocrinol. 2016;175(4):26571.

19. Kim LN, Kwon HK, Moon HI, et al. Sonography of the median nerve in carpal tunnel syndrome with diabetic neuropathy. Am J Phys Med Rehabil. 2014;93:897-907.

20. Klauser AS, Halpern EJ, De Zordo T, Feuchtner GM, Arora R, Gruber J, Martinoli C, Löscher WN. Carpal tunnel syndrome assessment with US: value of additional cross-sectional area measurements of the median nerve in patients versus healthy volunteers. Radiology. 2009 Jan;250(1):171-7.

21. England JD, Gronseth GS, Franklin G, et al. Distal symmetric polyneuropathy: a definition for clinical research: report of the American Academy of Neurology, the American Association of Electrodiagnostic Medicine, and the American Academy of physical medicine and rehabilitation. Neurology. 2005;64:199-207.

22. Gazioglu S, Boz C, Cakmak VA. Electrodiagnosis of carpal tunnel syndrome in patients with diabetic polyneuropathy. Clin Neurophysiol. 2011;122:14639.

23. Kwon HK, Hwang M, Yoon DW. Frequency and severity of carpal tunnel syndrome according to level of cervical radiculopathy: double crush syndrome? Clin Neurophysiol. 2006;117:1256-9.

24. Kwon HK, Kim L, Park YK, et al. Frequency of carpal tunnel syndrome acoording to the severity of diabetic neuropathy. J Korean Acad Rehabil Med. 2005;29:272-5.

25. Padua L, LoMonaco M, Gregori B, Valente EM, Padua R, Tonali $\mathrm{P}$. Neurophysiological classification and sensitivity in 500 carpal tunnel syndrome hands. Acta Neurol Scand. 1997;96(4):211-7.

26. Hammer HB, Hovden IA, Haavardsholm EA, Kvien TK. Ultrasonography shows increased cross-sectional area of the median nerve in patients with arthritis and carpal tunnel syndrome. Rheumatology (Oxford). 2006;45(5): 584-8.

27. Seror P. Sonography and electrodiagnosis in carpal tunnel syndrome diagnosis: an analysis of the literature. Eur J Radiol. 2008;67(1):146-52.

28. Möller I, Miguel M, Bong D, Zaottini F, Martinoli C. The peripheral nerves: An update on ultrasound and magneticresonance imaging. Clin Exp Rheumatol. 2018;36 Suppl 114(5):145-58.

29. Ibrahim I, Khan W, Goddard N, Smitham P. Carpal tunnel syndrome: a review of the recent literature. Open Orthop J. 2012;6(6):69.

30. Baiee RH, NJ AL-M, Al-Rubiae SJ, Hammoodi ZH, Abass FN. Neurophysiological findings in patients with carpal tunnel syndrome by nerve conduction study in comparing with ultrasound study. J Nat Sci Res. 2015;5(1):9.

31. Kataruka M, Pramanik R, Das P, Haldar RN, Samanta S, Bhakat D. The role of musculoskeletal US as diagnostic tool. IJPMR. 2014;25(2):44-9.

32. Mhoon JT, Juel VC, Hobson-Webb LD. Median nerve ultrasound as a screening tool in carpal tunnel syndrome: correlation of cross sectional area measures with electrophysiologic abnormality. Muscle Nerve. 2012;46:86170.

33. Mohammadi A, Afshar A, Etemadi A, Masoudi S, Baghizadeh A. Diagnostic value of cross-sectional area of median nerve in grading severity of carpal tunnel syndrome. Arch Iran Med. 2010;13(6):516-21.

34. Tsai N-W, Lee L-H, Huang C-R, Chang W-N, Wang H-C, Lin Y-J, Lin W-C, Lin T-K, Cheng B-C, Yu-Jih S, Kung C-T, Chen S-F. The diagnostic value of ultrasonography in carpal tunnel syndrome: a comparison between diabetic and non-diabetic patients. BMC Neurol. 2013;13:65

35. Lee $\mathrm{CH}$, Choi H, Yoon JS, Kang S. Carpal tunnel syndrome assessment with ultrasonography: a comparison between non-diabetic and diabetic patients. Ann Rehabil Med. 2018;42(1):85-91.

36. Lundborg G, Gelberman RH, Minteer-Convery M, Lee YF, Hargens AR. Median nerve compression in the carpal tunnel: functional response to experimentally induced controlled pressure. J Hand Surg Am. 1982;7(3):2529.

37. Thomsen NO, Mojaddidi M, Malik RA, Dahlin LB. Reduced myelinated nerve fibre and endoneurial capillary densities in the forearm of diabetic and nondiabetic patients with carpal tunnel syndrome. Acta Neuropathol. 2009;118: 785-91.

\section{Publisher's Note}

Springer Nature remains neutral with regard to jurisdictional claims in published maps and institutional affiliations.
Ready to submit your research? Choose BMC and benefit from:

- fast, convenient online submission

- thorough peer review by experienced researchers in your field

- rapid publication on acceptance

- support for research data, including large and complex data types

- gold Open Access which fosters wider collaboration and increased citations

- maximum visibility for your research: over $100 \mathrm{M}$ website views per year

At BMC, research is always in progress.

Learn more biomedcentral.com/submissions 